\title{
Susceptibilité au cancer colique et instabilité de I'ADN : un nouveau gène localisé en 2p15-16
}

Hier encore, deux grandes classes de gènes impliqués dans le processus oncogénique pouvaient être distinguées : les proto-oncogènes, dont certaines mutations entraînent une activation leur conférant un rôle dominant sur la genèse tumorale, et les anti-oncogènes ou gènes suppresseurs de tumeur, pour lesquels les deux allèles doivent être inactivés pour être à l'origine d'un processus tumoral. Le gène APC, par exemple, fait partie de cette deuxième catégorie et confère une prédisposition aux cancers du côlon, traduite cliniquement par une polypose adénomateuse familiale (FAP) précédant environ $1 \%$ des cancers coliques.

Une étude de liaison vient de révéler un nouveau gène impliqué dans les formes familiales du cancer du côlon (ou syndrome de Lynch) et qui semble agir selon une voie inconnue jusqu'alors. Les équipes de B. Vogelstein (Baltimore, MA, USA) et de A. de la Chapelle (Helsinki, Finlande) ont en effet étudié les membres de deux familles très étendues - l'une, originaire d'Amérique du Nord et l'autre, de Nouvelle-Zélande - , dont certains étaient porteurs de cancers coliques sans polypose héréditaire [1, 2]. Les auteurs ont ainsi recherché un lien entre un locus chromosomique donné et la survenue de l'affection en utilisant 345 marqueurs très informatifs, de type microsatellite, établis par J. Weissenbach (Généthon, Ivry). Une liaison a été obtenue avec un marqueur localisé sur le bras court du chromosome 2 (2p15-16).

La démarche logique faisant suite à cette observation fut de rechercher, chez les patients atteints de cancers coliques, une perte d'hétérozygotie qui aurait fait du gène responsable de la susceptibilité à ce cancer un potentiel anti-oncogène. La perte d'hétérozygotie correspond en effet généralement à une délétion d'un des deux allèles d'un anti-oncogène et rend alors le sujet qui en est porteur plus susceptible au développement d'une tumeur par mutation et inactivation du deuxième allèle. Aucune délétion ne fut dépistée dans cette région du chromosome 2. En revanche, l'analyse de l'ADN tumoral révéla une variation de longueur du microsatellite en question comparée à la taille obtenue dans l'ADN d'organes sains des mêmes patients et entre les différentes tumeurs. En réalité, l'équipe américano-finlandaise ainsi que deux autres équipes américaines (Rochester, MI et La Jolla, CA, USA) $[3,4]$, révèlent que cette hétérogénéité de longueur de l'ADN n'est pas restreinte à ce microsatellite. Elle est également retrouvée au niveau des microsatellites situés sur d'autres chromosomes dans 12 à $28 \%$ des tumeurs coliques familiales ou sporadiques analysées. De plus, il semble que cette instabilité des microsatellites définisse une entité tumorale particulière sur le plan de la localisation (nette prédominance d'atteinte de la portion proximale du côlon) et du pronostic (moindre gravité).

La nature du gène impliqué et la relation de cause à effet entre cette instabilité de l'ADN et le processus oncogénique restent encore à définir.
Les auteurs suggèrent que ce gène code pour un facteur nécessaire à la réplication de l'ADN. Ainsi, une mutation somatique de ce gène pourrait entraîner des altérations géniques disséminées sur l'ensemble du génome d'une cellule, dont certaines pourraient être tumorigènes.

En attendant confirmation éventuelle de ces hypothèses, il est d'ores et déjà possible d'identifier, grâce au marqueur du chromosome 2 , les sujets à risque d'une famille qui compte des membres atteints d'un cancer colique sans polypose préalable.

H. G.

1. Peltomaki P, Aaltonen LA, Sistonen P, Pylkkänen L, Mecklin JP, Järvinen H, Green JS, Jass JR, Weber JL, Leach FS, Petersen GM, Hamilton SR, de la Chapelle A, Vogelstein B. Genetic mapping of a locus predisposing to human colorectal cancer. Science 1993 ; $260: 810-2$

2. Aaltonen LA, Peltomaki $P$, Leach FS, Sistonen P, Pylkkänen L, Mecklin JP, Järvinen H, Powell SM, Jen J, Hamilton SR, Petersen $G M$, Kinzler $K W$, Vogelstein $B$, de la Chapelle A. Clues to the pathogenesis of familial colorectal cancer. Science 1993 ; 260 : 812-6. 3. Thibodeau SN, Bren G, Schaid D. Microsatellite instability in cancer of the proximal colon. Science 1993 ; 260 : 816-9.

4. Ionov Y, Peinado MA, Malkhosyar S, Shibata D, Perucho $M$. Ubiquitous somatic mutations in simple repeated sequences reveal a new mechanism for colonic carcinogenesis. Nature 1993 ; 363 : 558-561 